\title{
Spatial clustering of habitat structure effects patterns of community composition and diversity
}

\author{
Florian Altermatt ${ }^{1,2,3}$ and Marcel Holyoak ${ }^{2}$ \\ ${ }^{1}$ Department of Aquatic Ecology, Eawag: Swiss Federal Institute of Aquatic Science and Technology, Überlandstrasse 133, \\ CH-8600 Dübendorf, Switzerland \\ ${ }^{2}$ Department of Environmental Science and Policy, University of California, 1 Shields Avenue, Davis, California 95616 USA
}

\begin{abstract}
Natural ecosystems often show highly productive habitats that are clustered in space. Environmental disturbances are also often nonrandomly distributed in space and are either intrinsically linked to habitat quality or independent in occurrence. Theoretical studies predict that configuration and aggregation of habitat patch quality and disturbances can affect metacommunity composition and diversity, but experimental evidence is largely lacking. In a metacommunity experiment, we tested the effects of spatially autocorrelated disturbance and spatial aggregation of patch quality on regional and local richness, among-community dissimilarity, and community composition. We found that spatial aggregation of patch quality generally increased among-community dissimilarity (based on two measures of $\beta$ diversity) of communities containing protozoa and rotifers in microcosms. There were significant interacting effects of landscape structure and location of disturbances on $\beta$ diversity, which depended in part on the specific $\beta$ diversity measures used. Effects of disturbance on composition and richness in aggregated landscapes were generally dependent on distance and connectivity among habitat patches of different types. Our results also show that effects of disturbances in single patches cannot directly be extrapolated to the landscape scale: the predictions may be correct when only species richness is considered, but important changes in $\beta$ diversity may be overlooked. There is a need for biodiversity and conservation studies to consider the spatial aggregation of habitat quality and disturbance, as well as connectivity among spatial aggregations.
\end{abstract}

Key words: beta diversity; density; dispersal; metacommunity; microcosm; protozoa; spatial autocorrelation.

\section{INTRODUCTION}

A major goal of ecologists is to understand how environmental and biotic factors affect and shape community composition at different spatial scales. Resource availability, patch size, habitat connectivity, and disturbances are seen as major players defining the composition of natural communities. Theoretical studies have extensively addressed the significance of these factors individually. We know that resource availability affects competitive interactions (Tilman 1980, Chesson 2000), that larger patches have higher species diversity (MacArthur and Wilson 1967), and that intermediate connectivity and disturbance at intermediate frequency may allow the coexistence of species that would otherwise be out-competed (Levin and Paine 1974, Connell 1978, Chesson and Warner 1981, Loreau and Mouquet 1999). By contrast, comparative studies conducted in natural communities found that individual environmental factors often do not accurately and fully explain community composition and diversity (e.g.,

Manuscript received 1 July 2011; revised 16 December 2011; accepted 20 December 2011. Corresponding Editor: N. J. Gotelli.

${ }^{3}$ E-mail: florian.altermatt@eawag.ch
Pollock et al. 1998, Genner et al. 2004). It is intriguing that island biogeography factors such as habitat area and isolation can be surprisingly poor predictors of species occupancy in natural communities (Condit et al. 2002, Prugh et al. 2008). Also, the pure effect of spatial distance and environmental heterogeneity only partially contributes to explaining overall diversity (Sousa 1979, Forbes and Chase 2002, Tews et al. 2004). Unexplained variance is due to other factors, such as unmeasured spatial structure and interactions between environmental factors (Kallimanis et al. 2005, Van Buskirk 2005, Ramette and Tiedje 2007).

A frequently overlooked factor is that many studies were done in a nonspatial context or in simple homogeneous or randomly arranged landscapes. In natural landscapes, however, patches of different quality are often clustered or nonrandomly arranged along gradients. For example, water depth and light availability radially change from the center to the outside of coral reefs (Grigg 1983). Furthermore, environmental disturbances are often nonrandom in distribution (Sousa 1984), and may even be intrinsically linked to habitat patch quality. For example, in riparian areas, soil depth and humidity depend on the distance to the linear watercourse (Dudgeon et al. 2006), as does the 


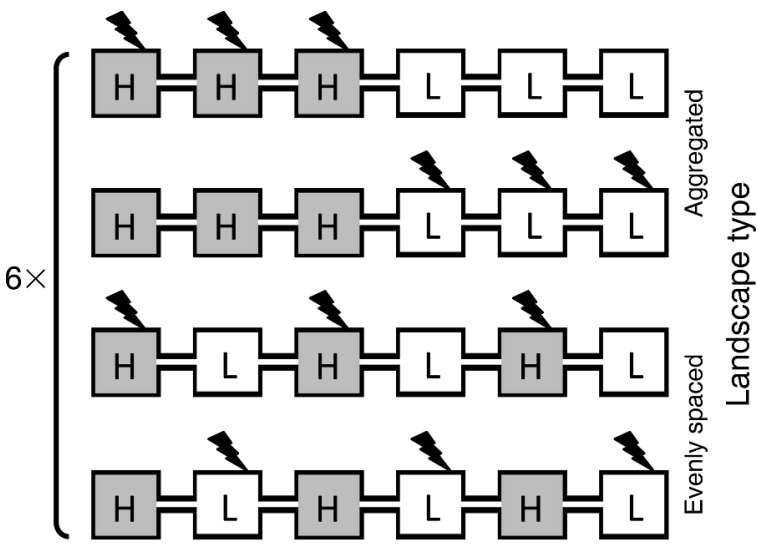

$\square$ High-nutrient patch $\square$ Low-nutrient patch $>$ Disturbance

FIG. 1. Setup of the microcosm experiment. Each of the 24 landscapes consisted of a metacommunity with six linearly arranged patches. Patches were connected by tubing through which individuals could actively disperse. In half of the landscapes, patches of high and low quality (respective nutrient levels) were spatially aggregated, while in the other half of the landscapes they were evenly spaced. We applied two coinciding disturbance treatments, in which either all three patches of high or low quality were disturbed at regular intervals. Disturbance (indicated by the lightening bolt) consisted of density-independent mortality.

magnitude of flood disturbances that are spatially linked to distance from the river bed and elevation (Pollock et al. 1998). In mountain ranges, alternating north- and south-facing slopes create a spatially regular pattern in habitat patches that differ in soil depth, snow cover, and climate (Körner 2003). This geological structure also affects the occurrence of disturbances such as fires, avalanches, and rockslides (Sousa 1984). Spatial autocorrelation of habitat quality is thought to be beneficial for diversity and species persistence (Kallimanis et al. 2005). The effect, however, may not be universal (Forbes and Chase 2002) and may also depend on the type of dispersal. In contrast, there is good theoretical and empirical support that spatial autocorrelation of disturbances has detrimental effects on local communities (Vuilleumier et al. 2007, Elkin and Possingham 2008), and that dispersal distance and directionality in such landscapes defines recovery from disturbances (Altermatt et al. 2011b). Coral reefs, riparian habitats, or mountainous habitats not only exhibit specific spatial arrangements of patch quality and disturbances, but are also diversity hotspots (Connell 1978, Körner 2003, Dudgeon et al. 2006, Vorosmarty et al. 2010). Thus, the understanding of factors affecting and creating high diversity is of general interest for the understanding and maintenance of global diversity.

Recent theoretical studies have considered real-world complexities like nonrandom distributions of habitat patches of different qualities and interactions of environmental factors at a variety of spatial scales (Vuilleumier et al. 2007, Elkin and Possingham 2008, Büchi et al. 2009). Such studies found that configuration and aggregation of patch quality and nonrandom disturbances may affect metapopulation persistence or mean life-history traits of species (Vuilleumier et al. 2007, Elkin and Possingham 2008, Büchi et al. 2009). Importantly, the scale of spatial aggregation of disturbance often coincides with the scale of spatial aggregation of habitat quality. This is especially true for disturbances that are mechanistically coupled with the environmental factors related to habitat quality (Sousa 1984, Körner 2003, Dudgeon et al. 2006, Vorosmarty et al. 2010). Consequently, habitat quality and disturbance are likely to have strong combined effects on community composition. Despite this functional coupling, there is lack of experimental studies addressing how the coincidence of spatially aggregated habitat quality and disturbance affect diversity and community composition.

We experimentally examined the effect of spatially autocorrelated disturbance on regional and local species richness and among-community dissimilarity $(\gamma, \alpha$, and $\beta$ diversity) in landscapes where habitats of different quality were aggregated or evenly spaced (Fig. 1). Since measures of diversity, especially $\beta$ diversity, are manifold and the best practice of using them is still an ongoing discussion (Gotelli and Colwell 2001, Koleff et al. 2003, Jost 2007, Anderson et al. 2011), we used and compared methods that are based on presence-absence data with methods based on density (relative abundance) data. We specifically wanted to address two questions: (1) Do the effects on diversity patterns of spatial aggregation of disturbance interact with spatial aggregation of patch quality? (2) Are the effects of coincidence in aggregation in patch quality and disturbance synergistic or antagonistic for diversity?

To address our questions, we conducted a laboratory experiment in aquatic microcosms containing protozoan and rotifer species that used common freshwater bacteria as a food resource. The metacommunity experiment allowed the controlled manipulation of individual factors. Replicated landscapes consisted of six linearly arranged patches that were connected with tubing, allowing organisms to actively disperse between patches (Fig. 1). Half of the patches within a landscape were of high habitat quality (high nutrient level), while the other patches were of low habitat quality (low nutrient level). We constructed two different landscape types, in which the habitat patches of high and low quality were either spatially aggregated or evenly distributed. We applied repeated spatially autocorrelated disturbances that coincided with either patch quality type and measured persistence and density of species at the end of the multi-generation experiment.

\section{Material and Methods}

\section{Communities}

We conducted an experiment in aquatic microcosms containing seven protozoan species, one rotifer species, 
and a set of common freshwater bacteria as a food resource. Bacteria, in turn, were supported on a plantbased nutrient medium and decomposing wheat seeds. The seven protozoan species were Chilomonas sp., Colpidium sp., Euglena gracilis, Euplotes aediculatus, Paramecium aurelia, Paramecium bursaria, and Spirostomum sp., and the rotifer species was Cephalodella sp. (see Plate 1). Five of the protozoans and the rotifer we studied were originally collected from a single pond (McGrady-Steed et al. 1997), while Chilomonas sp. and Spirostomum sp. came from Carolina Biological Supply Company (Burlington, North Carolina, USA). All species are bacterivores, although Eug. gracilis, Eup. aediculatus, and $P$. bursaria can also photosynthesize. Some of the larger protozoans (e.g., Spirostomum) and the rotifer not only feed on bacteria but may also prey on smaller protozoans.

\section{Setup of the landscapes}

We used metacommunities consisting of six patches per landscape (Fig. 1), as well as isolated control patches. Like in Davies et al. (2009), each of the individual patches consisted of a $125-\mathrm{mL}$ Nalgene square polycarbonate wide-mouth bottle. The six bottles of a metacommunity were linearly connected with $11 \mathrm{~cm}$ long segments of silicon tubing (inner diameter $6 \mathrm{~mm}$ ). To control for effects of tubing such as spatial refuge or spatial heterogeneity, we furnished the isolated controls with equal length of tubing but clamped off the center of the tubing. All bottles were loosely capped throughout the experiment to minimize evaporation, and sterile technique was used. Protozoans and rotifers could actively disperse between the patches through the tubing (Altermatt et al. 2011a). Three patches within a landscape were of high habitat quality, while the other three patches were of low habitat quality (based on the availability of nutrients). Patches of high and low habitat quality (i.e., high and low nutrient levels) were either spatially aggregated or evenly spaced (Fig. 1). Following previous work (Haddad et al. 2008), we used different nutrient concentrations to manipulate habitat quality. Patches of high habitat quality contained 100 $\mathrm{mL}$ of nutrient medium and two autoclaved wheat seeds as an additional carbon source for the bacteria. The medium was made from a standard soil-water solution, prepared by mixing $2.4 \mathrm{~g}$ of sterilized soil and $0.6875 \mathrm{~g}$ of Protozoan Pellet (Carolina Biological Supply) in 1.5 $\mathrm{L}$ of spring water and then sterilized by steam autoclaving. Patches of low habitat quality received medium which was diluted 1:9 with autoclaved spring water, and no wheat seeds. A day before adding protozoa, each patch was inoculated with a mixed bacterial culture (Bacillus cereus, B. subtilis, and Serratia marcescens obtained from Carolina Biological Supply) to provide resources for protozoans. We had 12 isolated controls of high and 12 of low habitat quality, six of both experienced the same disturbance treatment as in the main experiment.
Each patch was initiated with a community of all eight protozoa or rotifer species. Initial population numbers per patch were about 100 individuals for all species but Spirostomum, which naturally occurs at lower densities than the other species and was initiated with a population of about 60 individuals per bottle. Initial population numbers were set to avoid extinction caused by demographic stochasticity before application of the first disturbance treatment. After one week, about 100 individuals of three species ( $P$. aurelia, P. bursaria, and Eug. gracilis) were added to all patches to ensure establishment. All additions were done by adding a small inoculum of medium containing the individuals, and an equivalent volume of medium was removed from each patch prior to addition. All communities were allowed to grow for 13 days before disturbance treatments were applied.

\section{Disturbances}

For each landscape type (aggregated and evenly spaced) we had two disturbance treatments, each replicated six times (i.e., in total 24 landscapes). In one disturbance treatment, all three high-quality patches were disturbed, while in the other disturbance treatment, all three low-quality patches were disturbed (Fig. 1). Disturbance consisted of replacement of $99 \%$ of the bottle contents with sterilized media (high or low nutrient concentration, respectively). Similar disturbance treatments were used in earlier studies (Haddad et al. 2008, Altermatt et al. 2011b). Disturbances did not affect the $\sim 4 \mathrm{~mL}$ of medium in the tubing of the dispersal corridor, in which protozoa could survive, just as dispersing individuals in a habitat matrix would not experience patch-specific disturbance in nature. Disturbance occurred every four days over a period of 20 days. After three disturbance events, we replaced $10 \mathrm{~mL}$ of medium in all patches to avoid deteriorations due to accumulation of waste products.

\section{Density measures}

We estimated the density (and presence/absence) of the eight protozoa and rotifer species in each replicate with a stereo microscope (20-40× magnification) 35 days after the initiation of the experiment. Samples were taken two days after the last disturbance events. We thoroughly mixed the contents of each bottle prior to the sampling. Because of the different species' sizes and densities, volumes censused were species-specifically adjusted to obtain an adequate density estimate (for details, see Haddad et al. 2008, Davies et al. 2009). In short, we measured density for small species and/or species at high density in a sample of $0.5-1.0 \mathrm{~mL}$, which we diluted if the species was too abundant to be counted accurately. For large species or species at lower density, we screened up to $10 \mathrm{~mL}$ of medium. In preceding trials, we confirmed that our point of decision of a species' absence was robust and unlikely to produce false absence estimates, independent of the density and 


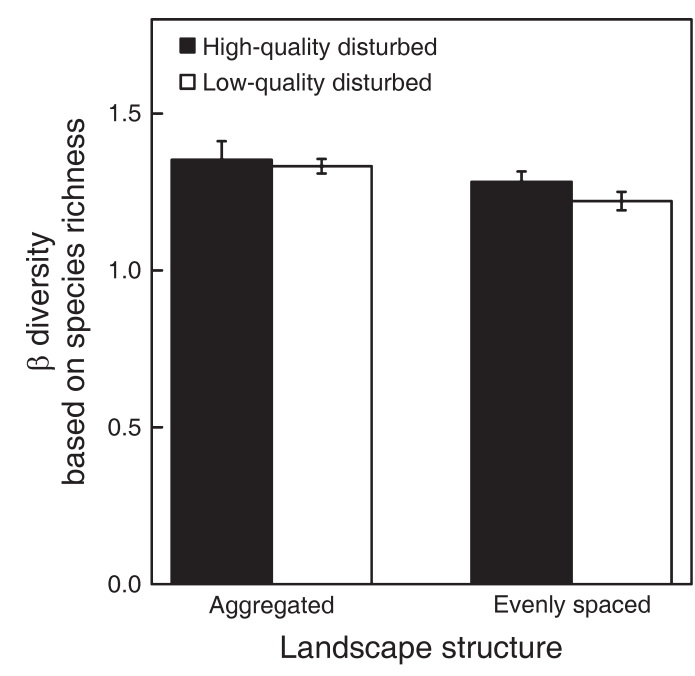

FIG. 2. $\quad \beta$ diversity (mean $\pm \mathrm{SE}$ ) based on species richness (i.e., presence-absence data; Hill number with exponent $q=0$ ), describing dissimilarity among communities within landscapes in response to landscape structure (aggregated vs. evenly spaced) and coinciding disturbance of high-quality or lowquality patches (black and white bars, respectively). We calculated $\beta$ diversity independently for each of the six replicated landscapes of a treatment combination.

presence of other species. Since we were working with a closed system (i.e., the maximum number of species was given by our initial communities that were created in patches of a fixed volume), we knew the upper maximum of species that could be present. By measuring density and presence/absence for each species individually and using a common maximum volume sampled for each species, we avoided a bias in diversity measures that often arise in samples from natural systems due to different sample sizes (Jost 2007). In other words, sampling of species individually meant that diversity patterns were not influenced by the sampling effort, and rarefaction is not needed to control for sampling effort. Our "complete" knowledge on the communities allowed us to use diversity measures that include abundance information with diversity measures that only use presence/absence data, and that may provide complementary information about diversity patterns ( $\mathrm{R}$ Development Core Team 2010; vegan package available online). ${ }^{4}$ One landscape (aggregated, low-nutrient patches disturbed) was lost from the experiment because of leaking, reducing our total number of landscapes to 23 .

\section{Statistical analyses}

All analyses were conducted in $\mathrm{R}$ ( $\mathrm{R}$ Development Core Team 2010) using the library vegan (see footnote $2)$. We calculated for each landscape the total species richness $(\gamma$ diversity), mean local species richness $(\alpha$ diversity), and among-community dissimilarity ( $\beta$ diver-

\footnotetext{
${ }^{4}$ http://cran.r-project.org/web/packages/vegan/index.html
}

sity). We then calculated mean values of these estimates for each landscape. We used Hill numbers with an exponent of $q=0$ and $q=1$ (Jost 2007) to calculate measures of $\beta$ diversity (Anderson et al. 2011). The first $\beta$ diversity measure is based on species richness alone, while the second $\beta$ diversity measure is an exponentiated Shannon's index that weighs all species by their frequency (Jost 2007, Anderson et al. 2011). The exponent of Shannon's index $(q=1)$ is the "true order of $q$ " in Jost's (2007) terminology. We finally compared the observed $\beta$ diversity (Hill number with order $q=1$ ) from the four different landscape types with $\beta$ diversity values that we calculated from the observed species densities in isolated controls. We bootstrapped $\beta$ diversity values (10000 times) for the "virtual landscapes" that were put together in the same four landscape configurations as the real landscapes with respect to connectivity and disturbance of high- vs. lowquality patches. The bootstrapped values allowed us to see if connectivity and spatial arrangement had a significant effect on $\beta$ diversity or not compared to landscapes without dispersal.

We conducted all of our analyses on the landscape level, that is, we had 23 independent measures for the statistical analyses. We used ANOVAs to analyze the effect of landscape structure (aggregated vs. evenly spaced) and disturbance of high quality or low quality patches as well as their interaction on our response variables. Residual deviances of models were used as the goodness-of-fit criterion in the evaluation of the models.

\section{RESUlts AND DisCUSSION}

Spatial aggregation of patch quality and the coincidence of disturbance (Fig. 1) had strong and significant effects on $\beta$ diversity and community composition in our experimental metacommunities (Figs. 2 and 3). The $\beta$ diversity based on presence-absence data (Hill number with $q=0$; Jost 2007) was significantly higher in the aggregated landscapes than in the evenly distributed landscapes (ANOVA, $F_{1,19}=5.2, P=0.04$; Fig. 2). For $\beta$ diversity based on presence-absence data, there was no significant difference between disturbances that affected either high or low quality patches $\left(F_{1,19}=1.1, P=0.31\right)$, and also no significant interaction between landscape structure and disturbance $\left(F_{1,19}=0.26, P=0.62\right)$. When incorporating density data, $\beta$ diversity (Hill number with $q=1$; Jost 2007) was significantly affected by an interaction of landscape type and the spatial coincidence of disturbance (ANOVA, $F_{1,19}=7.9, P=0.01$; Fig. 3). Furthermore, $\beta$ diversity was significantly higher than other treatments when disturbances affected highquality patches $\left(F_{1,19}=18.4, P=0.0004\right)$. The influence of landscape structure was not significant in the main effect $\left(F_{1,19}=0.006, P=0.94\right)$.

It is notable that the observed effect of landscape structure on $\beta$ diversity depended on the specific $\beta$ diversity measure (based on presence-absence vs. density data), and resulted in a qualitatively different 
interpretation. Presence-absence data are the most frequent form of data to which $\beta$ diversity measures are applied (Koleff et al. 2003). However, in our protozoa system, as well as in many natural systems, it is relevant to consider density because it can vary over several orders of magnitudes between species (Altermatt et al. 2011a), and depends on the local environmental conditions (Fig. A2). A possible explanation for the discrepancy in the relative order of how disturbance of high vs. low quality patches affected the two $\beta$ diversity measures may be due to the different effects of disturbances on population densities vs. extinctions. Likely, density of species is more affected by the quality of the patches (Haddad et al. 2008), while species richness alone was affected more by the spatial distribution of patch types and connectivity that compensated extinctions by colonizations. This is supported by the large range of densities observed among and within species (Appendix: Fig. A2), and corresponds to the effects of disturbance and nutrients on density observed in other studies (Haddad et al. 2008, Altermatt et al. 2011a). The effect of spatial pattern and connectivity on species richness has been demonstrated in previous studies (Cadotte 2006), while the herein demonstrated effects on community dissimilarity have received little attention. Our work highlights that a comprehensive description of diversities is necessary for a comprehensive understanding of metacommunities. It also confirms a tenet of the metacommunity concept, that communities of interacting species interconnected by dispersal may create non-trivial interactions (Wilson 1992, Leibold et al. 2004), and be another source of variation that promotes biodiversity.

Species depending on high-nutrient patches but not resistant to disturbances suffered the strongest decreases in population size and were at highest risk of extinction (Appendix: Figs. A1 and A2) when the patches were disturbed. However, recovery from disturbances also depended on patch position relative to the closest undisturbed patch (Fig. A1), which caused the aforementioned strong effect of landscape structure on $\beta$ diversity. These findings fit with our previous experiments in which connectivity counteracted the negative effect of disturbances on local populations through mass-effect-driven movement of individuals from undisturbed to disturbed patches (Altermatt et al. 2011a). The distance-dependent effect observed here is consistent with such mass effects. The recovery of local communities through post-disturbance dispersal depended on landscape structure, and this altered the interaction between landscape structure and disturbance recovery. Similar distance-dependent post-disturbance recovery has been noted for local species diversity in treefall gaps in lowland tropical forests (Norden et al. 2009), but both the effects for $\beta$ diversity and experimental manipulation of disturbance and landscape structure are more novel.

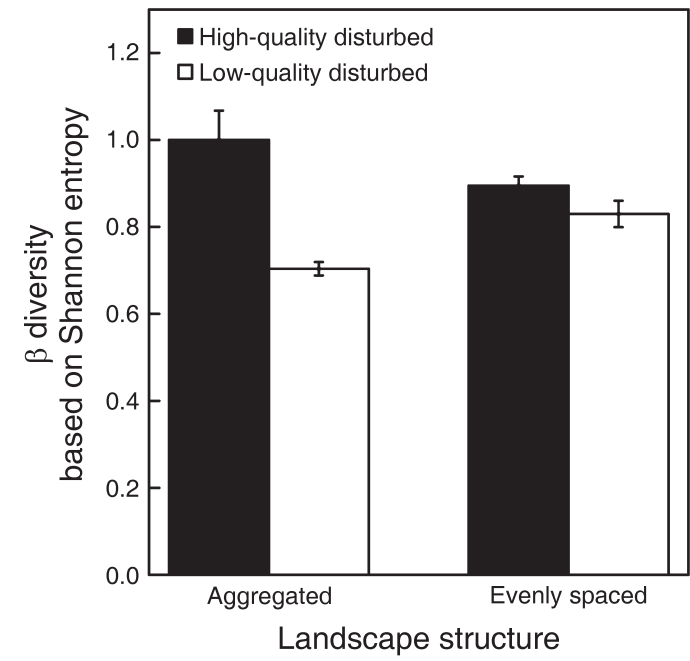

FIG. 3. The $\beta$ diversity (mean $\pm \mathrm{SE}$ ) based on relative abundance of species (i.e., Shannon entropy; Hill number with exponent $q=1$ ), describing dissimilarity among communities within landscapes in response to landscape structure (aggregated vs. evenly spaced) and coinciding disturbance of high-quality or low-quality patches (black and white bars, respectively). We calculated $\beta$ diversity independently for each of the six replicated landscapes of a treatment combination.

We compared our observed differences in $\beta$ diversity based on density data (Fig. 3) among the four different types of landscapes (Fig. 1) with bootstrapped $\beta$ diversity values from "virtual landscapes" simulated using the density and diversity values of isolated controls. We found that two out of the four landscape types had significantly different $\beta$ diversity values than one would expect from the landscapes constructed from the isolated controls (Appendix: Fig. A3). Aggregated landscapes in which the low quality patches were disturbed had a significantly lower $\beta$ diversity than expected from the "virtual landscapes" $(P=0.048)$, while evenly spaced landscapes in which the low quality patches were disturbed had a significantly higher $\beta$ diversity $(P=0.05)$. These findings support our above finding that connectivity, dispersal among patches and the specific layout of patch quality had a significant effect on community composition and $\beta$ diversity. In the two other types of landscapes, the observed $\beta$ diversity was not significantly different from the "virtual landscapes" $(P>0.1)$, suggesting that $\beta$ diversity was more driven by local dynamics and less by dispersal.

By contrast to $\beta$ diversity, there was neither a significant effect of landscape structure on regional species richness at the landscape level $(\gamma$ diversity. ANOVA, $F_{1,19}=0.01, P=0.9$; Appendix: Fig. A4) nor on local species richness ( $\alpha$ diversity. $F_{1,19}=3.5, P=$ 0.08 , Fig. 4). As expected, $\gamma$ and $\alpha$ diversity were significantly lower in landscapes in which the high quality patches were disturbed compared to landscapes 


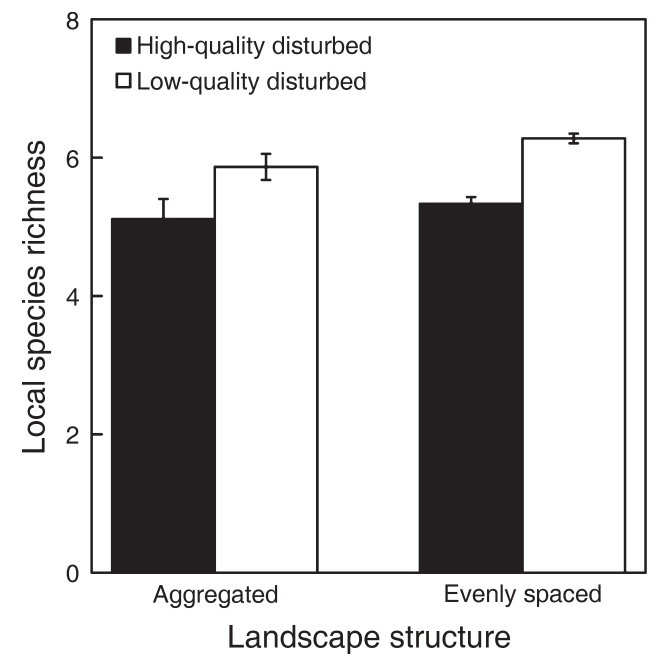

FIG. 4. Local species richness (mean $\pm \mathrm{SE}$ ) in response to landscape structure (aggregated vs. evenly spaced) and disturbance of high-quality or low-quality patches (black and white bars, respectively). For each of the six replicated landscapes of a treatment combination, mean local species richness of protozoa and rotifer species (i.e., mean number of species within each patch) was calculated independently.

in which the low quality patches were disturbed $(\gamma$ diversity, $F_{1,19}=23.0, P=0.0001 ; \alpha$ diversity, ANOVA, $\left.F_{1,19}=20.8, P=0.0002\right)$. Both for $\gamma$ and $\alpha$ diversity, the interaction term between landscape structure and disturbance was non-significant $\left(\gamma\right.$ diversity, $F_{1,19}=$ $0.13, P=0.73 ; \alpha$ diversity, $\left.F_{1,19}=0.25, P=0.62\right)$. The lower $\gamma$ diversity in landscapes in which the high quality patches were disturbed was mostly driven by a consistent extinction of one species (Spirostomum), which is not very tolerant to disturbances and is a bad competitor in low nutrient environments (Haddad et al. 2008). Being a bad disperser on top of that (Altermatt et al. 2011a) prevented recolonization of low-nutrient patches in the replicates where the high quality patches were undisturbed. Even though all patches within a landscape were connected, the dispersal ability of this species was below the minimal threshold necessary to exploit some favorable patches that were regionally available. As a conservation implication, it suggests that some species (e.g., large, slow growing and less mobile species) are less suitable to be protected in a metapopulation context and may require larger habitats rather than more patches. Our results indicate that the spatial arrangement of habitat quality may have no direct role in determining species richness with both passive dispersal (Forbes and Chase 2002) and active dispersal. Thus, by looking only at species richness, we would (falsely) conclude that landscape structure combined with spatial aggregation of disturbance did not affect communities. This also refutes the traditional focus on how disturbances and connectivity affect mean species richness. More generally, $\beta$ diversity may be of equal or greater importance to overall biodiversity (Loreau 2000, Anderson et al. 2011), especially given that we expect regional processes to maintain diversity within fragmented habitats. $\beta$ diversity is also central to concepts about what controls diversity in ecological communities. In one view, species composition is controlled by local environmental site characteristics that vary within the landscape. In such a scenario, $\beta$ diversity reflects deterministic processes, such as species' adaptations to differences in resource availability or resistance to disturbances (Bray and Curtis 1957, Hutchinson 1957, Whittaker 2001, Legendre et al. 2005). In another view, local species composition results from limited dispersal often coupled with speciation. The local community is thus a response to spatially limited dispersal history (Hubbell 2001, Condit et al. 2002, Muneepeerakul et al. 2008) and local species composition fluctuates in a random, autocorrelated way.

Our control populations in isolated patches helped to better understand the causality behind our findings. From previous work we knew that the species used in our experiment can coexist over many generations when kept in a favorable environment (Haddad et al. 2008). This was confirmed by the isolated controls with high nutrient level and no disturbances (Appendix: Fig. A5). In these controls, species richness was both significantly influenced by the nutrient level and disturbance: low nutrient patches had on average 2.1 fewer species per patch than high nutrient patches $\left(F_{1,19}=39.0, P<0.0001\right)$ and disturbed patches had on average 0.9 fewer species than undisturbed patches of the same nutrient level $\left(F_{1,19}=6.7, P=0.018\right)$. There was no significant interaction between nutrient level and disturbance $\left(F_{1,19}=0.23, P=0.64\right)$. Thus, the observed diversity differences in landscapes with different spatial layouts were direct consequences of dispersal among patches. In a previous study on the effects of recurrent disturbance on species diversity and density in two-patch metacommunities (Altermatt et al. 2011a), we separated the role of between-patch movement from subsequent population growth. We inferred that population recovery from disturbances largely depended on the influx of individuals from undisturbed patches, and subsequent population growth. The latter was directly linked to species-specific intrinsic growth rates (Altermatt et al. 2011a). Thereby, dispersal between neighboring patches interacts directly with the negative effects of disturbance. The current experiment shows that the effects of dispersal are not only distance dependent, but also limited to specific local habitat quality. A comparative study of forest patches in the tropics suggested the importance of such distance-dependent recovery dynamics, such that resilience depended not only on high levels of dispersal, but also on local presence of old-growth forest remnants (Norden et al. 2009). In our experiment, recovery of species richness from disturbances in aggregated 


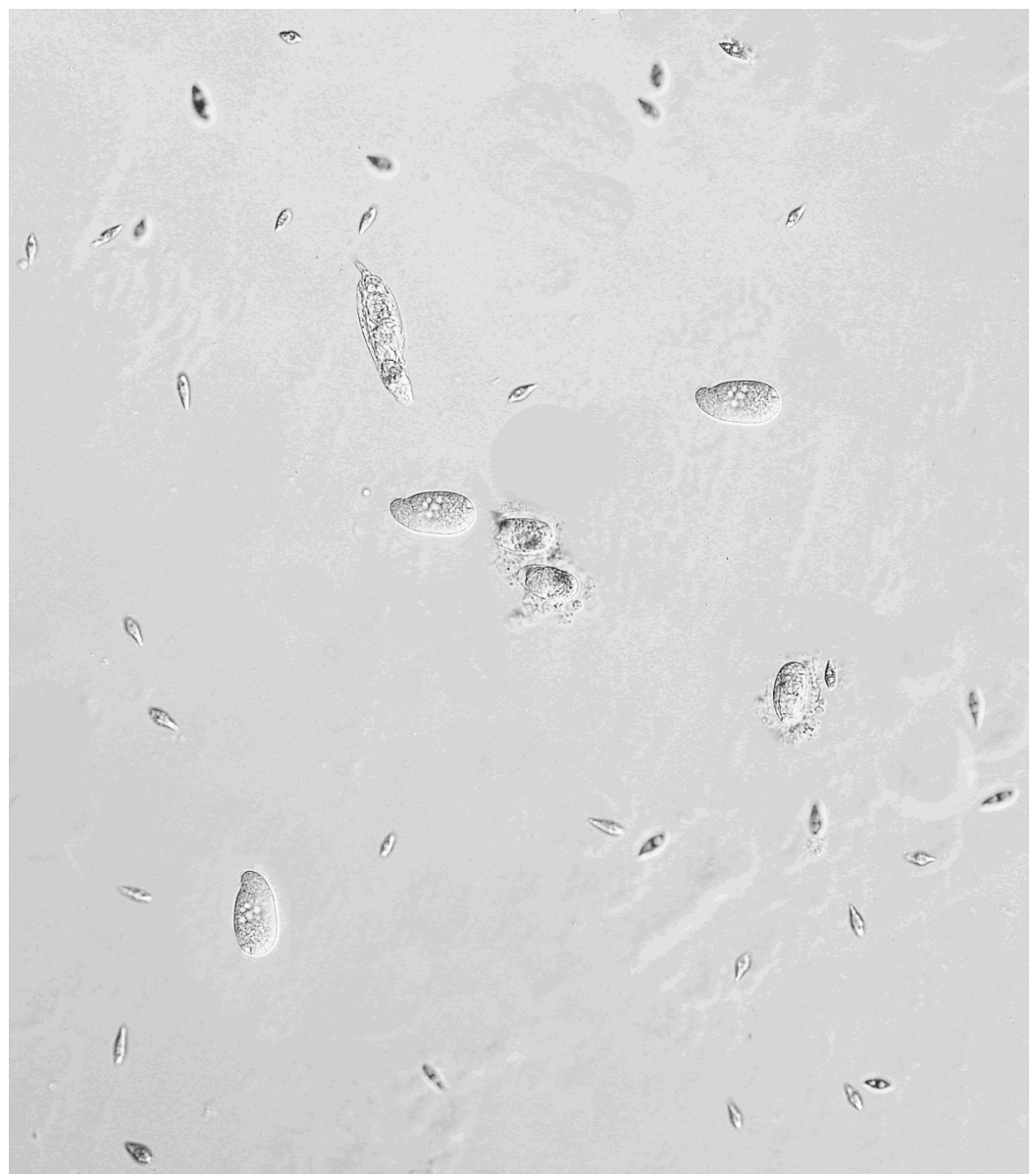

Plate 1. Light microscopy image showing two of the protists and the rotifer species used in the microcosm experiments. The depicted species are Colpidium sp., Euglena gracilis, and Cephalodella sp. Actual sizes of the species are about $80 \mu \mathrm{m}, 40 \mu \mathrm{m}$, and 110 $\mu \mathrm{m}$ respectively. The image was finished using digital image software, and some individuals were duplicated for visual reasons. Photo credits: Regula Illi and F. Altermatt.

patches depended, at least in landscapes in which the low-quality patches were disturbed, on the distance to the high-quality patches (Fig. A1). The negative effect of disturbances occurring every 3-10 generations (depending on the species) were frequent enough to prohibit a stepping-stone movement between neighboring patches that were disturbed from more distant, undisturbed patches. Thus, dispersal limitation directly caused differences in diversity, creating significantly different $\beta$ diversity patterns in differently structured landscapes.

Our results highlight that spatial autocorrelation of environmental variables can have strong and complex effects on spatially explicit diversity measures, such as $\beta$ diversity, while at the same time local and regional diversity measures were not affected. With these findings we support the idea that diversity measures at different scales do not necessarily react in the same way. It is known that there are no theoretical grounds for the belief that species interactions set an absolute upper limit to diversity at any scale (Loreau 2000). Loreau
(2000) also argued that saturation of local-regional richness curves does not tell us anything about community saturation due to species interactions, but is more fundamentally related to the scale at which a local community is defined. Consequently, a more promising challenge is to understand the relationship between $\alpha$ and $\beta$ diversity at multiple scales (Jost 2007), and the processes that determine it (Harrison and Cornell 2008). Identifying the relationship between the $\alpha$ and $\beta$ components of diversity at multiple scales may improve our understanding of the processes that control diversity over the whole range of scales. A further aspect explaining the observed non-trivial relationship among different levels of diversity may be that in our system, perhaps similar to various natural systems, many species can coexist in local communities (Hutchinson 1961, Haddad et al. 2008). When transient, highly dispersive, rare species contribute to local diversity, we would expect more predictable relationships between $\alpha, \beta$, and $\gamma$ diversity (Palardy and Witman 2011). Quite likely, the relationship between $\alpha$ and $\beta$ diversity would also be 
more predictable in a system in which the species are mostly affected by local competitive exclusion, and less by dispersal and their ability to recover from disturbance.

The principles highlighted here have important implications. Anthropogenic land conversion forces us to decide which habitat patches to prioritize for protection. This decision also depends on the intent of conservation measures: do we want to maximize regional species diversity, local species diversity, or among-community dissimilarity? How the spatial patterning of habitats influences community dynamics has been elusive for a long time. In conservation biology, usually the habitat patches of the best quality or those with the least likelihood to be disturbed are prioritized for protection. Our concerns and efforts should differ especially when disturbances are correlated with patch quality, and when the connectivity of patches is considered. Specifically, we think of cases when the patches of different quality are aggregated or evenly spaced. Here, we found strong effects at the level of community composition, using a manipulative experiment in which cause and effect can be determined. From a conservation perspective, protecting evenly spaced patches of a specific habitat type may maximize mean $\alpha$ diversity level at the landscape level, but comes at the cost of lower $\beta$ diversity. Community structuring processes are important in the context of on-going climate change. The likelihood and magnitude of disturbances are increasing (Min et al. 2011, Pall et al. 2011), and the spatial patterns of where disturbances are occurring are shifting. The resilience of natural communities may depend not only on their spatial arrangement and mean diversity, but even more on among-community dissimilarity. Such differences in $\beta$ diversity may be caused by disturbance and climate change (Altermatt et al. 2008). At the same time, under climate change, the spatially aggregated disturbances may shift, which can be detrimental since the spatial location of habitats of high quality and diversity is often fixed (nature reserves, national parks, etc.). Consequently, a better understanding of the interactive effects of spatial structure of the environment and disturbance on $\beta$ diversity is a high priority.

To conclude, our experiments showed that the spatial aggregation of disturbance and habitat quality had an interacting effect on community composition and diversity. This emphasizes the need to consider spatial distribution of habitat patches of different quality. Spatial scale should also not be overlooked. While we found effects at the spatial scales we looked at, it is yet to be determined more generally how the scale (grain) of landscape variation influences how disturbance and patch quality interact.

\section{ACKNOWLEDGMENTS}

We thank Annette Bieger for help with the experimental work and two anonymous reviewers for their helpful comments. F. Altermatt was supported by the Swiss National Science Foundation (grant no. PBBSP3-124435).

\section{Literature Cited}

Altermatt, F., A. Bieger, F. Carrara, A. Rinaldo, and M. Holyoak. 2011a. Effects of connectivity and recurrent local disturbances on community structure and population density in experimental metacommunities. PLoS ONE 6:e19525.

Altermatt, F., V. I. Pajunen, and D. Ebert. 2008. Climate change affects colonisation dynamics in a metacommunity of three Daphnia species. Global Change Biology 14:1209-1220.

Altermatt, F., S. Schreiber, and M. Holyoak. 2011b. Interactive effects of disturbance and dispersal directionality on species richness and composition in metacommunities. Ecology 92:859-870.

Anderson, M. J., et al. 2011. Navigating the multiple meanings of $\beta$ diversity: a roadmap for the practicing ecologist. Ecology Letters 14:19-28.

Bray, R. J., and J. T. Curtis. 1957. An ordination of the upland forest communities of southern Wisconsin. Ecological Monographs 27:325-349.

Büchi, L., P.-A. Christin, and A. H. Hirzel. 2009. The influence of environmental spatial structure on the life-history traits and diversity of species in a metacommunity. Ecological Modelling 220:2857-2864.

Cadotte, M. 2006. Metacommunity influences on community richness at multiple spatial scales: a microcosm experiment. Ecology 87:1008-1016.

Chesson, P. 2000. Mechanisms of maintenance of species diversity. Annual Review of Ecology and Systematics 31:343-366.

Chesson, P., and R. Warner. 1981. Environmental variability promotes coexistence in lottery competitive systems. American Naturalist 117:923-943.

Condit, R., et al. 2002. Beta-diversity in tropical forest trees. Science 295:666-669.

Connell, J. H. 1978. Diversity in tropical rainforests and coral reefs. Science 199:1302-1310.

Davies, K. F., M. Holyoak, K. A. Preston, V. A. Offeman, and Q. Lum. 2009. Factors controlling community structure in heterogeneous metacommunities. Journal of Animal Ecology 78:937-944.

Dudgeon, D., A. H. Arthington, M. O. Gessner, Z. I. Kawabata, D. J. Knowler, C. Lévêque, R. J. Naiman, A. H. Prieur-Richard, D. Soto, M. L. J. Stiassny, and C. A. Sullivan. 2006. Freshwater biodiversity: importance, threats, status and conservation challenges. Biological Reviews 81:163-182.

Elkin, C. M., and H. Possingham. 2008. The role of landscapedependent disturbance and dispersal in metapopulation persistence. American Naturalist 172:563-575.

Forbes, A. E., and J. N. Chase. 2002. The role of habitat connectivity and landscape geometry in experimental zooplankton metacommunities. Oikos 96:433-440.

Genner, M. J., M. I. Taylor, D. F. R. Cleary, S. J. Hawkins, M. E. Knight, and G. F. Turner. 2004. Beta diversity of rockrestricted cichlid fishes in Lake Malawi: importance of environmental and spatial factors. Ecography 27:601-610.

Gotelli, N. J., and R. K. Colwell. 2001. Quantifying biodiversity: procedures and pitfalls in the measurement and comparison of species richness. Ecology Letters 4:379-391.

Grigg, R. W. 1983. Community structure, succession and development of coral reefs in Hawaii. Marine Ecology Progress Series 11:1-14.

Haddad, N. M., M. Holyoak, T. M. Mata, K. F. Davies, B. A. Melbourne, and K. Preston. 2008. Species' traits predict the effects of disturbance and productivity on diversity. Ecology Letters 11:348-356. 
Harrison, S., and H. Cornell. 2008. Toward a better understanding of the regional causes of local community richness. Ecology Letters 11:969-979.

Hubbell, S. P. 2001. The unified neutral theory of biodiversity and biogeography. Princeton University Press, Princeton, New Jersey, USA.

Hutchinson, G. E. 1957. Concluding remarks. Cold Spring Harbor Symposium on Quantitative Biology 22:415-427.

Hutchinson, G. E. 1961. The paradox of the plankton. American Naturalist 95:137-145.

Jost, L. 2007. Partitioning diversity into independent alpha and beta components. Ecology 88:2427-2439.

Kallimanis, A. S., W. E. Kunin, J. M. Halley, and S. P. Sgardelis. 2005. Metapopulation extinction risk under spatially autocorrelated disturbance. Conservation Biology 19:534-546.

Koleff, P., K. J. Gaston, and J. J. Lennon. 2003. Measuring beta diversity for presence-absence data. Journal of Animal Ecology 72:367-382.

Körner, C. 2003. Alpine plant life - functional plant ecology of high mountain ecosystems. Springer, Heidelberg, Germany.

Legendre, P., D. Borcard, and P. R. Peres-Neto. 2005 Analyzing beta diversity: partitioning the spatial variation of community composition data. Ecological Monographs 75:435-450.

Leibold, M. A., et al. 2004. The metacommunity concept: a framework for multi-scale community ecology. Ecology Letters 7:601-613.

Levin, S. A., and R. T. Paine. 1974. Disturbance, patch formation, and community structure. Proceedings of the National Academy of Sciences USA 71:2744-2747.

Loreau, M. 2000. Are communities saturated? On the relationship between alpha, beta and gamma diversity. Ecology Letters 3:73-76.

Loreau, M., and N. Mouquet. 1999. Immigration and the maintenance of local species diversity. American Naturalist 154:427-440.

MacArthur, R. H., and E. O. Wilson. 1967. The theory of island biogeography. Princeton University Press, Princeton, New Jersey, USA.

McGrady-Steed, J., P. M. Harris, and P. J. Morin. 1997. Biodiversity regulates ecosystem predictability. Nature 390:162-165.

Min, S.-K., X. Zhang, F. W. Zwiers, and G. C. Hegerl. 2011. Human contribution to more-intense precipitation extremes. Nature 470:378-381.

Muneepeerakul, R., E. Bertuzzo, H. J. Lynch, W. F. Fagan, A. Rinaldo, and I. Rodriguez-Iturbe. 2008. Neutral metacommunity models predict fish diversity patterns in MississippiMissouri basin. Nature 453:220-222.

Norden, N., R. L. Chazdon, A. Chao, Y.-H. Jiang, and B. Vílchez-Alvarado. 2009. Resilience of tropical rain forests: tree community reassembly in secondary forests. Ecology Letters 12:385-394.
Palardy, J. E., and J. D. Witman. 2011. Water flow drives biodiversity by mediating rarity in marine benthic communities. Ecology Letters 14:63-68.

Pall, P., T. Aina, D. A. Stone, P. A. Stott, T. Nozawa, A. G. J. Hilberts, D. Lohmann, and M. R. Allen. 2011. Anthropogenic greenhouse gas contribution to flood risk in England and Wales in autumn 2000. Nature 470:382-385.

Pollock, M. M., R. J. Naiman, and T. A. Hanley. 1998. Plant species richness in riparian wetlands: a test of biodiversity theory. Ecology 79:94-105.

Prugh, L. R., K. E. Hodges, A. R. E. Sinclair, and J. S. Brashares. 2008. Effect of habitat area and isolation on fragmented animal populations. Proceedings of the National Academy of Science USA 105:20770-20775.

R Development Core Team. 2010. R: a language and environment for statistical computing. Version 2.12.1. R Foundation for Statistical Computing, Vienna, Austria. www.r-project.org

Ramette, A., and J. M. Tiedje. 2007. Multiscale responses of microbial life to spatial distance and environmental heterogeneity in a patchy ecosystem. Proceedings of the National Academy of Sciences USA 104:2761-2766.

Sousa, W. P. 1979. Experimental investigations of disturbance and ecological succession in a rocky inter-tidal algal community. Ecological Monographs 49:227-254

Sousa, W. P. 1984. The role of disturbances in natural communities. Annual Review of Ecology and Systematics 15:353-392.

Tews, J., U. Brose, V. Grimm, K. Tielborger, M. C. Wichmann, M. Schwager, and F. Jeltsch. 2004. Animal species diversity driven by habitat heterogeneity/diversity: the importance of keystone structures. Journal of Biogeography 31:79-92.

Tilman, D. 1980. Resources: a graphical-mechanistic approach to competition and predation. American Naturalist 116:362393.

Van Buskirk, J. 2005. Local and landscape influence on amphibian occurrence and abundance. Ecology 86:19361947.

Vorosmarty, C. J., P. B. McIntyre, M. O. Gessner, D. Dudgeon, A. Prusevich, P. Green, S. Glidden, S. E. Bunn, C. A. Sullivan, C. R. Liermann, and P. M. Davies. 2010. Global threats to human water security and river biodiversity. Nature 467:555-561.

Vuilleumier, S., C. Wilcox, B. J. Cairns, and H. P. Possingham. 2007. How patch configuration affects the impact of disturbances on metapopulation persistence. Theoretical Population Biology 72:77-85.

Whittaker, J. B. 2001. Insects and plants in a changing atmosphere. Journal of Ecology 89:507-518.

Wilson, D. S. 1992. Complex interactions in metacommunities, with implications for biodiversity and higher levels of selection. Ecology 73:1984-2000.

\section{Supplemental Material}

\section{Appendix}

Figures showing additional results and further diversity measures, especially for $\gamma$ and $\alpha$ diversity (Ecological Archives E093-097A1). 